This item was submitted to Loughborough's Research Repository by the author.

Items in Figshare are protected by copyright, with all rights reserved, unless otherwise indicated.

\title{
Violation of adiabaticity in magnetic billiards due to separatrix crossings
}

PLEASE CITE THE PUBLISHED VERSION

http://dx.doi.org/10.1063/1.4928473

PUBLISHER

(c) AIP Publishing LLC

VERSION

VoR (Version of Record)

PUBLISHER STATEMENT

This work is made available according to the conditions of the Creative Commons Attribution 3.0 Unported (CC BY 3.0) licence. Full details of this licence are available at: http://creativecommons.org/licenses/by/3.0/

\section{LICENCE}

CC BY 3.0

\section{REPOSITORY RECORD}

Artemyev, A.V., and Anatoly Neishtadt. 2019. "Violation of Adiabaticity in Magnetic Billiards Due to Separatrix Crossings". figshare. https://hdl.handle.net/2134/18870. 


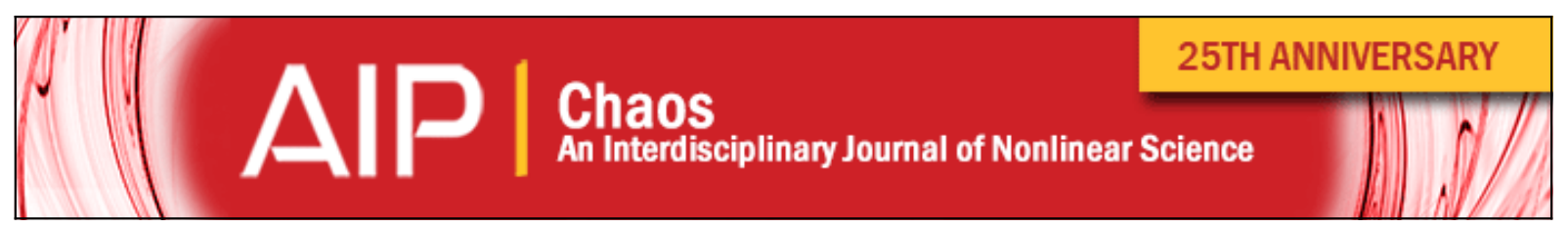

\section{Violation of adiabaticity in magnetic billiards due to separatrix crossings}

A. V. Artemyev and A. I. Neishtadt

Citation: Chaos 25, 083109 (2015); doi: 10.1063/1.4928473

View online: http://dx.doi.org/10.1063/1.4928473

View Table of Contents: http://scitation.aip.org/content/aip/journal/chaos/25/8?ver=pdfcov

Published by the AIP Publishing

\section{Articles you may be interested in}

Jumps of adiabatic invariant at the separatrix of a degenerate saddle point

Chaos 21, 043120 (2011); 10.1063/1.3657916

Adiabatic bistable evolution of dynamical systems governed by a Hamiltonian with separatrix crossing

Phys. Plasmas 13, 054502 (2006); 10.1063/1.2201927

Stickiness in mushroom billiards

Chaos 15, 033105 (2005); 10.1063/1.1979211

Semidispersing billiards with an infinite cusp. II

Chaos 13, 105 (2003); 10.1063/1.1539802

Convergence of Hamiltonian systems to billiards

Chaos 8, 466 (1998); 10.1063/1.166328

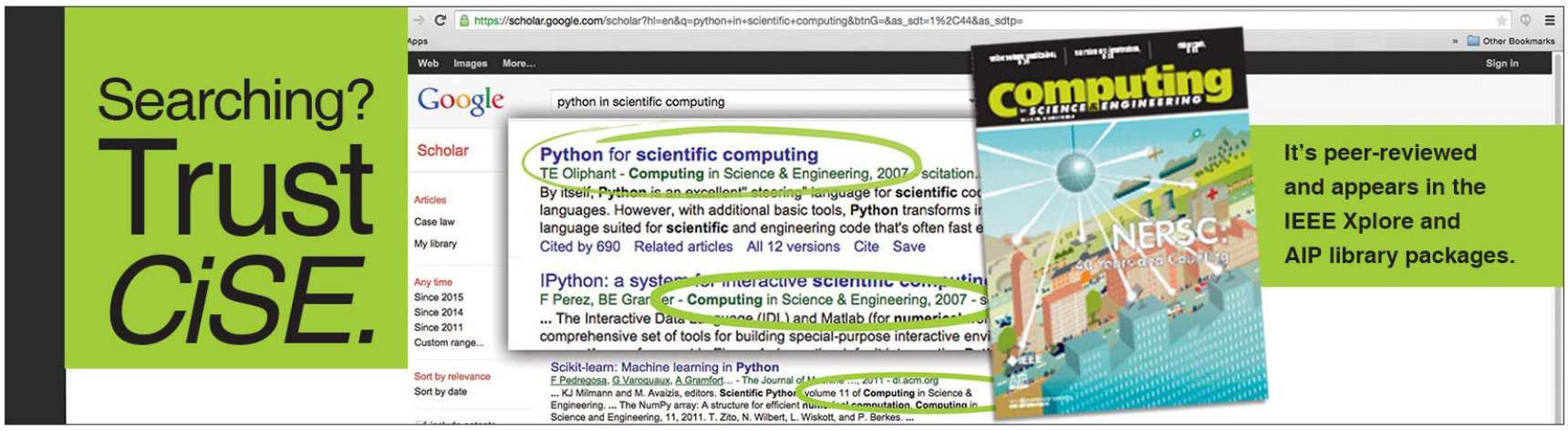




\title{
Violation of adiabaticity in magnetic billiards due to separatrix crossings
}

\author{
A. V. Artemyev ${ }^{1, a)}$ and A. I. Neishtadt ${ }^{1,2}$ \\ ${ }^{1}$ Space Research Institute, RAS, Profsouznaya St., 84/32, GSP-7, 117997 Moscow, Russia \\ ${ }^{2}$ Department of Mathematical Sciences, Loughborough University, LE11 3TU Loughborough, United Kingdom
}

(Received 8 April 2015; accepted 31 July 2015; published online 13 August 2015)

\begin{abstract}
We consider dynamics of magnetic billiards with curved boundaries and strong inhomogeneous magnetic field. We investigate a violation of adiabaticity of charged particle motion in this system. The destruction of the adiabatic invariance is due to the change of type of the particle trajectory: particles can drift along the boundary reflecting from it or rotate around the magnetic field at some distance from the boundary without collisions with it. Trajectories of these two types are demarcated in the phase space by a separatrix. Crossings of the separatrix result in jumps of the adiabatic invariant. We derive an asymptotic formula for such a jump and demonstrate that an accumulation of these jumps leads to the destruction of the adiabatic invariance. (C) 2015 AIP Publishing LLC. [http://dx.doi.org/10.1063/1.4928473]
\end{abstract}

The magnetic billiard is a well known model in statistical physics and nonlinear dynamics. This model imitates many real complicated systems describing various problems in magnetism, plasma physics, solid state physics, and astrophysics. The destruction of adiabaticity of charged particle motion in magnetic billiards simulates such important phenomena as exponential decay of correlations and breaking of time reversal symmetry in dynamical systems. In this paper, we investigate a new mechanism responsible for the violation of adiabaticity for systems with impacts. This mechanism is related to the separatrix crossing in the phase space. We apply methods of the adiabatic theory of smooth systems to magnetic billiards (i.e., for systems with impacts) and derive the expression determining the rate of adiabatic invariance destruction.

\section{INTRODUCTION}

Billiards are considered as universal models of many physical processes (see special section devoted to billiard models ${ }^{27}$ and review Ref. 19). Although, these are rather simple systems with geometrical laws of particle motion, they are able to simulate various statistical properties, ${ }^{8,41}$ statistical relaxation processes, ${ }^{10}$ the property of exponential decay of correlations, ${ }^{12,13}$ ergodicity of gas models, ${ }^{6}$ and breaking of time reversal symmetry of motion. ${ }^{11}$ Billiard also serves as a simple model of the Fermi acceleration process. ${ }^{28,29,36}$

Among many billiard models, the model of magnetic billiards (i.e., motion of charged particles inside some plane domain with the imposed magnetic field ${ }^{39}$ ) plays an important role. The magnetic billiard serves as a useful model for quantum mechanical problems ${ }^{15,22,30}$ and modeling of magnetic properties of materials (diamagnetism, paramagnetism, conductance, magnetic edge state, etc.), ${ }^{1,20,26,31,42}$ interaction of matter with laser field, ${ }^{21,38}$ and molecular/atom dynamics. ${ }^{2,3,37}$

In the model of magnetic billiards, the magnetic field amplitude controls a regularity level of the billiard

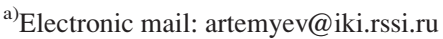

motion. ${ }^{7,16}$ There are several investigations of stochastization of motion in the magnetic billiards with and without curvature of the boundaries of domain where charged particles move. ${ }^{18,23,43,44}$ One of possible triggers of stochastization of motion is the destruction of adiabatic invariance (for magnetic billiards, the role of this invariant is played by the classical magnetic moment ${ }^{35}$ ). It was shown that destruction of the adiabaticity in magnetic billiards can realize in case of an inhomogeneous magnetic field. ${ }^{34}$ The primary origin of this process is related to jumps of the adiabatic invariant due to the change of type of particle motion (from drifting along the boundary to gyrorotation at some distance from the boundary without collisions). Different types of particle motion correspond to different domains in the phase space demarcated by a separatrix. There is a general theory of destruction of adiabatic invariance due to the separatrix crossing in smooth systems.9,32,33,46 Recently, this theory was generalized for systems with elastic impacts. ${ }^{17}$ In this paper, we adopt this theory to derive the equation for the rate of the adiabatic invariance destruction in magnetic billiards with an inhomogeneous magnetic field.

\section{GENERAL EQUATIONS}

We apply the Hamiltonian approach for investigation of charged particle motion in system with impacts (see also Refs. 49 and 50). This motion inside the 2D domain near the curved boundary and under the action of the transverse magnetic field can be described using curved coordinates $s$ and $r$ : $s$ is directed along the boundary, while $r$ is the transverse coordinate (see scheme in Fig. 1).

The corresponding Hamiltonian and sympletic structure have the form ${ }^{34}$

$$
\begin{aligned}
H= & \frac{1}{2 m}\left(p_{r}^{2}+\frac{p_{s}^{2}}{(1+k(s) r)^{2}}\right) \\
\omega^{2}= & d p_{r} \wedge d r+d p_{s} \wedge d s \\
& +\varepsilon^{-1}(1+k(s) r) \frac{e}{c} B(r, s) d r \wedge d s .
\end{aligned}
$$



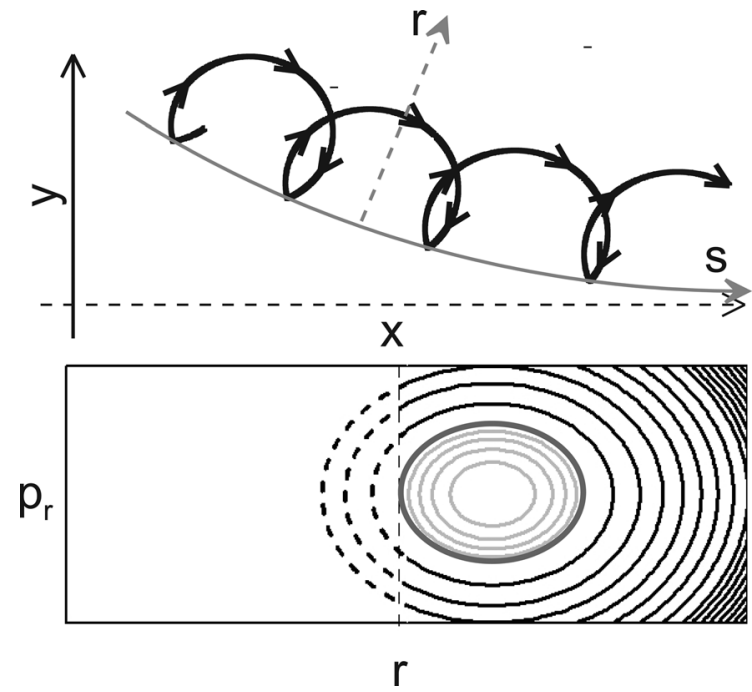

FIG. 1. Top panel shows the fragment of the particle trajectory, while the bottom panel shows the corresponding phase portrait in the $\left(r, p_{r}\right)$ plane.

Here, $p_{r}$ and $p_{s}$ are components of particle momentum, $e$ and $c$ are particle charge and speed of light, $\varepsilon^{-1} B$ is the magnetic field magnitude, $k(s)$ is the boundary curvature, and $\varepsilon$ is a constant positive parameter. We consider the charged particle motion in the strong magnetic field, i.e., $\varepsilon \ll 1$ and amplitude of $B$ is of the order of one. The reflection from the boundary $r=0$ corresponds to the transformation $p_{s} \rightarrow p_{s}$, $p_{r} \rightarrow-p_{r}$.

We introduce a canonical momentum $P_{s}=p_{s}+\varepsilon^{-1}$ $A(r, s)$ where

$$
A(r, s)=\frac{e}{c} \int_{0}^{r}(1+k(s) \eta) B(\eta, s) d \eta .
$$

Thus, Hamiltonian (1) takes the form

$$
\begin{aligned}
H & =\frac{1}{2 m}\left(p_{r}^{2}+\left(\frac{P_{s}-\varepsilon^{-1} A(r, s)}{1+k(s) r}\right)^{2}\right), \\
\omega^{2} & =d p_{r} \wedge d r+d P_{s} \wedge d s .
\end{aligned}
$$

We expand Eq. (2) up to terms $\sim r^{2}$

$$
A(r, s) \approx \frac{e}{c} B_{0}(s) r+\frac{e}{2 c} r^{2}\left(k(s) B_{0}(s)+B_{0}^{\prime}\right),
$$

where $B_{0}=B(0, s)$ and $B_{0}^{\prime}=\partial B /\left.\partial r\right|_{r=0}$. We introduce new variables $r / \varepsilon \rightarrow r, s / \varepsilon \rightarrow s, t / \varepsilon \rightarrow t$ and expand Hamiltonian (3) as

$$
\begin{aligned}
H & =H_{0}\left(p_{r}, r, \varepsilon s, P_{s}\right)+\varepsilon H_{1}\left(r, \varepsilon s, P_{s}\right) \\
H_{0} & =\frac{1}{2 m} p_{r}^{2}+\frac{1}{2 m} U \\
U & =\left(P_{s}-\frac{e}{c} B_{0}(\varepsilon s) r\right)^{2}, \\
H_{1} & =-\frac{1}{m} U k(\varepsilon s) r-\frac{e}{2 c m} \sqrt{U} r^{2}\left(k(\varepsilon s) B_{0}(\varepsilon s)+B_{0}^{\prime}(\varepsilon s)\right) .
\end{aligned}
$$

We introduce dimensionless variables and parameters

$$
\begin{aligned}
\left(p_{r}, P_{s}\right) & \rightarrow\left(p_{r}, P_{s}\right) / \sqrt{m h_{0}}, \\
b(\varepsilon s) & =B_{0}(\varepsilon s) / B_{0}(0), \\
s & \rightarrow s e B_{0}(0) / c \sqrt{m h_{0}}, \\
r & \rightarrow r e B_{0}(0) / c \sqrt{m h_{0}}, \\
k(\varepsilon s) & \rightarrow k(\varepsilon s) \sqrt{m h_{0}} c / e B_{0}(0), \\
t & \rightarrow \operatorname{te} B_{0}(0) / m c, \\
B_{0}^{\prime}(\varepsilon s) / B_{0}(0) & =b_{0}^{\prime} e B_{0}(0) / c \sqrt{m h_{0}}
\end{aligned}
$$

and rewrite Eq. (5) as

$$
\begin{aligned}
& H_{0}=\frac{1}{2} p_{r}^{2}+\frac{1}{2}\left(P_{s}-b r\right)^{2} \\
& H_{1}=-\left(P_{s}-b r\right)^{2} k r-\frac{1}{2}\left(P_{s}-b r\right) r^{2}\left(k b+b_{0}^{\prime}\right) .
\end{aligned}
$$

Here, $h_{0}$ is a characteristic value of particle energy, while dimensionless (normalized on $h_{0}$ ) energy is $h$. For frozen $\left(s, P_{s}\right)$ Hamiltonian $H_{0}$ describes periodic particle oscillations near the boundary $r=0$. The phase portrait shown in Fig. 1 demonstrates that there are two types of particle trajectories: with collisions and without collisions with the boundary. The phase region filled by trajectories of these two types are demarcated by the separatrix (shown by the solid grey curve). The region surrounded by the separatrix corresponds to particles that do not collide with the boundary. Particles colliding with the boundary $r=0$ are reflected from it. For slowly varying $\left(s, P_{s}\right)$, the position of the separatrix changes due to magnetic field inhomogeneity, and particles can change the type of motion, i.e., particles colliding with the boundary can start moving along the trajectories without collisions. Such separatrix crossing leads to violation of the adiabaticity of the motion because the period of particle oscillations near the separatrix depends on the particle energy singularly (cf. Ref. 17). To describe this violation of the adiabaticity we introduce the action as the area surrounded by the trajectory ${ }^{25}$ and describe the dynamics of this action

$$
\begin{aligned}
I & =\frac{1}{2 \pi} \oint p_{r} d r \\
& =\frac{1}{\pi} \int_{0}^{r_{\max }} \sqrt{2 h-\left(P_{s}-b(\varepsilon s) r\right)^{2}} d r,
\end{aligned}
$$

where the coordinate $r_{\max }$ is defined as

$$
r_{\max }=\frac{P_{s}+\sqrt{2 h}}{b(\varepsilon s)} .
$$

To introduce $I$ as a new variable we use the generating function

$$
W\left(r, \hat{I}, s, \hat{P}_{s}\right)=s \hat{P}_{s}+\int_{0}^{r} \sqrt{2 h-\left(P_{s}-b(\varepsilon s) \tilde{r}\right)^{2}} d \tilde{r},
$$

where the new momentum $\hat{P}_{s}=P_{s}-\partial W / \partial s$. Thus, the new Hamiltonian is 


$$
\begin{aligned}
\hat{H}= & \hat{H}_{0}\left(I, \varepsilon s, P_{s}\right)+\varepsilon \tilde{H}_{1}\left(I, \phi, \varepsilon s, P_{s}\right) \\
& +\varepsilon \hat{H}_{1}\left(I, \phi, \varepsilon s, P_{s}\right) \\
\tilde{H}_{1}= & \frac{\partial W}{\partial s} \frac{\partial H}{\partial P_{s}}-\frac{\partial H_{0}}{\partial s} \frac{\partial W}{\partial P_{s}}
\end{aligned}
$$

where $\hat{H}_{1}$ is $H_{1}$ from Eq. (7) written in new variables and we keep notation $I$ for $\hat{I}$. Instead of the variable conjugate to $I$, we use a phase $\phi$ defined as

$$
\begin{aligned}
r & =\frac{P_{s}+\sqrt{2 h} \sin (\phi)}{b(\varepsilon s)}, \\
\dot{\phi} & =b, \quad \phi \in\left[-\phi^{*}, \pi+\phi^{*}\right], \\
\phi^{*} & =\arcsin \left(P_{s} / \sqrt{2 h}\right),
\end{aligned}
$$

$\phi$ is a gyrophase, but it changes from one collision with the boundary to another one. In the new variables, the Hamiltonian $H_{1}$ takes the form

$$
\begin{aligned}
\hat{H}_{1}= & \frac{1}{2} \frac{(2 h)^{3 / 2}}{b^{2}}\left(\frac{P_{s}}{\sqrt{2 h}}\right)^{2}\left(b_{0}^{\prime}+k b\right) \sin (\phi) \\
& +\frac{1}{2} \frac{(2 h)^{3 / 2}}{b^{2}} 2 b_{0}^{\prime} \frac{P_{s}}{\sqrt{2 h}} \sin ^{2}(\phi) \\
& +\frac{1}{2} \frac{(2 h)^{3 / 2}}{b^{2}}\left(b_{0}^{\prime}-k b\right) \sin ^{3}(\phi) .
\end{aligned}
$$

Fig. 1 shows that for frozen values of $\left(s, P_{s}\right)$ particles move along the periodic trajectories in the phase plane $\left(r, p_{r}\right)$. For slowly changing $\left(s, P_{s}\right)$, the area surrounded by these trajectories and boundary $r=0$ can be considered as an adiabatically conserved quantity. This invariant is conserved with an accuracy $\sim \varepsilon$. However, we can introduce the improved adiabatic invariant $J$ which is conserved everywhere far from the separatrix with the accuracy $\sim \varepsilon^{2}$. The adiabatic invariant experiences small jumps in case of change of type of motion. Such change appears when a charged particle moving along the boundary (and reflecting from it) takes off from the boundary and starts drifting along the contours $B(r, s)=$ const. In analogy with smooth systems (without collisions, see Ref. 32), the change of type of motion could result in a jump of $I$ (see Refs. 17 and 34). In case when a particle comes to the boundary again after a certain time, jumps of the adiabatic invariant may result in stochastization of charged particle motion. ${ }^{34}$ To estimate the efficiency of this stochastization, one should derive the expression for the jump of the adiabatic invariant. To derive the expression for the jump, use the improved adiabatic invariant $J=I+\varepsilon u$ where

$$
u=\frac{\hat{H}_{1}+\tilde{H}_{1}-\left\langle\hat{H}_{1}+\tilde{H}_{1}\right\rangle}{\partial H_{0} / \partial I}-\frac{\partial I}{\partial P_{s}} \frac{\partial W}{\partial s}
$$

and brackets denote the averaging over $\phi$ oscillations. ${ }^{32}$ Function $u$ can be calculated at any point along the $r$-axis. For the point $r=0$ (i.e., at the boundary), Eq. (14) takes the form

$$
u=-\frac{\left\langle\hat{H}_{1}\right\rangle+\left\langle\tilde{H}_{1}\right\rangle}{\partial H_{0} / \partial I}=-\frac{T}{2 \pi}\left(\left\langle\hat{H}_{1}\right\rangle+\left\langle\tilde{H}_{1}\right\rangle\right)
$$

where

$$
\begin{aligned}
4 \pi\left\langle\tilde{H}_{1}\right\rangle= & \int_{0}^{T}\left(\frac{\partial H_{0}}{\partial P_{s}} \int_{0}^{t} \frac{\partial H_{0}}{\partial \varepsilon s} d \tilde{t}\right) d t \\
& -\int_{0}^{T}\left(\frac{\partial H_{0}}{\partial \varepsilon s} \int_{0}^{t} \frac{\partial H_{0}}{\partial P_{s}} d \tilde{t}\right) d t
\end{aligned}
$$

and $T$ is the period of particle oscillation in the $\left(r, p_{r}\right)$ plane (the time interval between two collisions with the boundary). Thus, to derive the expression for jump $\Delta J$, one should consider two terms: $\Delta I$ and $\Delta u$.

\section{JUMP OF THE ADIABATIC INVARIANT}

The main variation of the adiabatic invariant corresponds to particle motion near (and taking off from) the boundary. Thus, we consider the close vicinity of the moment of particle taking off from the boundary. This moment corresponds to the equation $P_{s}-\sqrt{2 h}=0$. Thus, following the general approach (see Ref. 32), we introduce a variable $K$ as a difference of particle energy $h$ and energy calculated at the separatrix $H_{\text {sep }}=P_{s}^{2} / 2: K=h-P_{s}^{2} / 2=h\left(1-P_{s}^{2} / 2 h\right)$. At the close vicinity of the boundary, $K$ is small (at the boundary $H=H_{\text {sep }}$ and $K=0$ ). To derive an expression for change of adiabatic invariant at time-scale of one period of oscillations at the $\left(r, p_{r}\right)$ plane, we need to obtain formula for period of these oscillations $T$ and rate of change $\varepsilon \Theta$ of area $S$ surrounded by the separatrix.

To obtain formula for $T$, we use the identity $T$ $=2 \pi(\partial I / \partial h)=2 \pi(\partial I / \partial K)$ and consider small enough $\kappa=K / h$ (i.e., we consider particle motion near the separatrix). We evaluate integral (8)

$$
\begin{aligned}
I & =\frac{2 h}{\pi b} \int_{-P_{s} / \sqrt{2 h}}^{1} \sqrt{1-y^{2}} d y \\
& =\frac{2 h}{\pi b} \frac{1}{2}\left(\frac{\pi}{2}+\frac{P_{s}}{\sqrt{2 h}} \sqrt{1-\left(P_{s} / \sqrt{2 h}\right)^{2}}+\arcsin \left(\frac{P_{s}}{\sqrt{2 h}}\right)\right) \\
& =\frac{2 h}{\pi b} \frac{1}{2}\left(\frac{\pi}{2}+\sqrt{1-\kappa} \sqrt{\kappa}+\arcsin (\sqrt{1-\kappa})\right)
\end{aligned}
$$

and expand it around $\kappa=0$

$$
I \approx \frac{2 h}{\pi b}\left(\frac{\pi}{2}-\frac{1}{3} \kappa^{3 / 2}\right) .
$$

Equation (19) provides the following expression for period of particle oscillations:

$$
T \approx T_{\text {sep }}-3 \pi a \sqrt{\kappa},
$$

where $T_{\text {sep }}$ is regular (not depending on $\kappa$ ) part of period (i.e., period calculated along the separatrix), while $a=2 /$ $(3 \pi b)$. 
The value of $\Theta$ can be calculated as $\Theta=\left\{S, H_{0}\right\}$ $=(\partial S / \partial \varepsilon s)\left(\partial H_{0} / \partial P_{s}\right)-\left(\partial S / P_{s}\right)\left(\partial H_{0} / \partial \varepsilon s\right)$. In the limit $\kappa$ $\rightarrow 0$, we have $\Theta \approx-\left(\partial S / \partial P_{s}\right)\left(\partial H_{0} / \partial \varepsilon s\right)$ where (see Ref. 32)

$$
\begin{aligned}
\frac{\partial H_{0}}{\partial \varepsilon s} & =-\frac{\partial I / \partial \varepsilon s}{\partial I / \partial h}=\frac{b_{s}^{\prime}}{b} h, \\
\frac{\partial S}{\partial P_{s}} & =-\oint \frac{\partial\left(H-H_{s e p}\right)}{\partial P_{s}} d t=b \oint r d t=\frac{2 \pi \sqrt{2 h}}{b} .
\end{aligned}
$$

Thus, we have $\varepsilon \Theta=-\pi b_{s}^{\prime}(2 h)^{3 / 2} / b^{2}$.

Then, the same procedure should be performed with $u$ term given by Eq. (15). For term $\left\langle\bar{H}_{1}\right\rangle$, we have

$$
\begin{aligned}
\left\langle\hat{H}_{1}\right\rangle= & \frac{1}{2} \frac{(2 h)^{3 / 2}}{b^{3} T} 2 b_{0}^{\prime} \frac{P_{s}}{\sqrt{2 h}} \int_{-\phi^{*}}^{\pi+\phi^{*}} \sin ^{2}(\phi) d \phi \\
& +\frac{1}{2} \frac{(2 h)^{3 / 2}}{b^{3} T}\left(b_{0}^{\prime}-k b\right) \int_{-\phi^{*}}^{\pi+\phi^{*}} \sin ^{3}(\phi) d \phi \\
& +\frac{1}{2} \frac{(2 h)^{3 / 2}}{b^{3} T}\left(\frac{P_{s}}{\sqrt{2 h}}\right)^{2}\left(b_{0}^{\prime}+k b\right) \int_{-\phi^{*}}^{\pi+\phi^{*}} \sin (\phi) d \phi \\
= & \frac{(2 h)^{3 / 2}}{b^{3} T}\left(\frac{P_{s}}{\sqrt{2 h}}\right)^{2}\left(b_{0}^{\prime}+k b\right) \cos \phi^{*} \\
& +\frac{(2 h)^{3 / 2}}{b^{3} T} b_{0}^{\prime} \frac{P_{s}}{\sqrt{2 h}}\left(\frac{\pi}{2}+\phi^{*}-\frac{1}{2} \sin 2 \phi^{*}\right) \\
& +\frac{(2 h)^{3 / 2}}{b^{3} T} \frac{2}{3}\left(b_{0}^{\prime}-k b\right)\left(\frac{1}{2} \sin ^{2} \phi^{*}+1\right) \cos \phi^{*},
\end{aligned}
$$

where $\phi^{*}=\arcsin (\sqrt{1-\kappa})$. Thus, expansion of Eq. (22) around $\kappa=0$ gives

$$
\begin{aligned}
\left\langle\hat{H}_{1}\right\rangle \approx & \frac{(2 h)^{3 / 2}}{b^{3} T}\left(b_{0}^{\prime}+k b\right) \sqrt{\kappa}(1-\kappa) \\
& +\frac{(2 h)^{3 / 2}}{b^{3} T} b_{0}^{\prime}\left(\pi-2 \sqrt{\kappa}-\frac{\pi \kappa}{2}\right) \\
& +\frac{(2 h)^{3 / 2}}{b^{3} T}\left(b_{0}^{\prime}-k b\right) \sqrt{\kappa}\left(1-\frac{1}{3} \kappa\right) \\
\approx & \frac{(2 h)^{3 / 2}}{b^{3} T} b_{0}^{\prime} \pi(1-\kappa / 2) .
\end{aligned}
$$

For the second term in Eq. (15), one can find

$$
\begin{aligned}
\left\langle\tilde{H}_{1}\right\rangle= & \frac{1}{4 \pi} \int_{0}^{T}\left(\frac{\partial H_{0}}{\partial P_{s}} \int_{0}^{t} \frac{\partial H_{0}}{\partial \varepsilon s} d \tilde{t}-\frac{\partial H_{0}}{\partial \varepsilon s} \int_{0}^{t} \frac{\partial H_{0}}{\partial P_{s}} d \tilde{t}\right) d t \\
= & \frac{(2 h)^{3 / 2} b_{s}^{\prime}}{4 \pi b^{3}} \int_{-\phi^{*}+\pi}^{\phi^{*}} \sin ^{2}(\phi) \int_{-\phi^{*}}^{\phi} \sin (\tilde{\phi}) d \tilde{\phi} d \phi-\frac{(2 h)^{3 / 2} b_{s}^{\prime}}{4 \pi b^{3}} \\
& \times \int_{-\phi^{*}}^{\phi^{*}+\pi} \sin (\phi)\left(\int_{-\phi^{*}}^{\phi} \sin ^{2}(\tilde{\phi}) d \tilde{\phi}\right) d \phi=0
\end{aligned}
$$

Thus, expression for $J$ around $\kappa=0$ takes the form

$$
\begin{aligned}
J & =I+\varepsilon u=I-\varepsilon T\left\langle\bar{H}_{1}\right\rangle \\
& =I-\varepsilon \frac{(2 h)^{3 / 2}}{2 b^{3}} b_{0}^{\prime}(1-\kappa / 2) .
\end{aligned}
$$

Let us estimate effects of variations of terms $I$ and $u$ on jump of the improved adiabatic invariant $J$. One can show that for $\kappa \gtrsim \varepsilon$ the change of $J$ for one period of the oscillations (i.e., between two collisions) is a value of order $\varepsilon^{3} / \kappa^{3 / 2}$. The value of $\kappa$ decays by $\varepsilon \Theta$ between two collisions. Thus, the change of $J$ when $\kappa$ decays from a value $\sim 1$ to a value $\sim \varepsilon$ is $\sim \varepsilon^{2} / \varepsilon^{1 / 2} \sim \varepsilon^{3 / 2}$. The change of $J$ when $\kappa$ decays from a value $\sim 1$ to a value $\kappa_{*} \gg \varepsilon$ is $\sim \varepsilon^{2} / \kappa_{*}^{1 / 2} \ll \varepsilon^{3 / 2}$. Thus, for calculation of asymptotic of change of $J$, it is enough to consider motion when $\kappa \leq \kappa *$. However, the change of $\varepsilon u$ for this motion is $\sim \varepsilon \kappa_{*}$ at most. Take $\varepsilon \ll \kappa_{*} \ll \varepsilon^{1 / 2}$. Then, the change of $\varepsilon u$ for this motion is $\ll \varepsilon^{3 / 2}$. Thus, we can neglect the variation of $u$ in our calculation of asymptotic of jump of $J$ and consider only variation of $I$ for motion in the domain of small $\kappa$.

For one period of oscillations in the $\left(r, p_{r}\right)$ plane, we can write the following expression for change of $2 \pi I=S$ $+h \int_{0}^{\kappa} T\left(h \kappa, s, P_{s}\right) d \kappa$ (change between $i$ th and $(i+1)$ th collisions with boundary; the index $i$ decreases as the particle moves to the position of taking off):

$$
2 \pi\left(I_{i}-I_{i+1}\right)=\Delta S_{i}+h \int_{\kappa_{i+1}}^{\kappa_{i}} T\left(H_{\text {sep }}+h \kappa, s, P_{s}\right) d \kappa,
$$

where $T$ is given by Eq. (20), while the change of the area surrounded by the separatrix $\Delta S$ is

$$
\Delta S_{i}=S_{i}-S_{i+1}=\varepsilon \Theta \frac{1}{2}\left(T_{i}+T_{i+1}\right)
$$

and $\varepsilon \Theta$ is a rate of $S$ variation calculated for the position of taking off, while $T_{i}$ is the period calculated in the $i$ th collisions with boundary. Substituting Eq. (20) to Eq. (26), we get

$$
\begin{aligned}
2 \pi\left(I_{i}-I_{i+1}\right)= & \varepsilon \Theta T_{\text {sep }}-\frac{3}{2} \varepsilon \Theta \pi a\left(\kappa_{i}^{1 / 2}+\kappa_{i+1}^{1 / 2}\right) \\
& +h \int_{\kappa_{i+1}}^{\kappa_{i}}\left(T_{\text {sep }}-3 \pi a \kappa^{1 / 2}\right) d \kappa \\
= & \varepsilon \Theta T_{\text {sep }}-\frac{3}{2} \varepsilon \Theta \pi a\left(\kappa_{i}^{1 / 2}+\kappa_{i+1}^{1 / 2}\right) \\
& +h T_{\text {sep }}\left(\kappa_{i}-\kappa_{i+1}\right)-2 \pi a h\left(\kappa_{i}^{3 / 2}-\kappa_{i+1}^{3 / 2}\right) .
\end{aligned}
$$

As $h\left(\kappa_{i}-\kappa_{i+1}\right)=-\varepsilon \Theta$ (see Ref. 4), Eq. (28) can be rewritten as

$$
I_{i}-I_{i+1}=-\frac{3}{4} \varepsilon \Theta a\left(\kappa_{i}^{1 / 2}+\kappa_{i+1}^{1 / 2}\right)-a h\left(\kappa_{i}^{3 / 2}-\kappa_{i+1}^{3 / 2}\right) .
$$

We calculate the sum of changes (29) starting from the period $i=N-1$ far from the separatrix and up to period $i=0$ (the last period before the separatrix crossing) 


$$
\begin{aligned}
I_{0}-I_{N}= & \sum_{i=0}^{N-1}\left(I_{i}-I_{i+1}\right)=-a h\left(\kappa_{0}^{3 / 2}-\kappa_{N}^{3 / 2}\right) \\
& -\frac{3}{2} \varepsilon \Theta a\left(\sum_{i=0}^{N} \kappa_{i}^{1 / 2}-\frac{1}{2} \kappa_{N}^{1 / 2}-\frac{1}{2} \kappa_{0}^{1 / 2}\right) .
\end{aligned}
$$

We also should add the last period of oscillations including the separatrix crossing

$$
\begin{aligned}
I_{-1}-I_{0} & =\frac{1}{2 \pi}\left(\Delta S_{-1}-h \int_{0}^{\kappa_{0}} T d \kappa-h \int_{\kappa_{-1}}^{0} T_{s e p} d \kappa\right) \\
& =-\frac{3}{4} \varepsilon \Theta a \kappa_{0}^{1 / 2}+a h \kappa_{0}^{3 / 2},
\end{aligned}
$$

where $T_{\text {sep }}$ is the regular part of the period, i.e., the first term in Eq. (20). The regular part of integral compensates the regular part of $\Delta S_{-1}$. Sum of Eq. (30) and (31) gives

$$
I_{-1}-I_{N}=-\frac{3}{2} \varepsilon \Theta a \sum_{i=0}^{N} \kappa_{i}^{1 / 2}+a h \kappa_{N}^{3 / 2}+\frac{3}{4} \varepsilon \Theta a \kappa_{N}^{1 / 2} .
$$

We introduce the new variable $\xi=h \kappa_{0} /(\varepsilon \Theta) \in(0,1)$ and use expression $h \kappa_{i}=h \kappa_{0}+i \varepsilon \Theta$ to rewrite Eq. (32)

$$
\begin{aligned}
I_{-1}-I_{N}= & -A(\varepsilon \Theta)^{3 / 2} \\
& \times\left(\frac{3}{2} \sum_{i=0}^{N}(\xi+i)^{1 / 2}-(\xi+N)^{3 / 2}-\frac{3}{4}(\xi+N)^{1 / 2}\right),
\end{aligned}
$$

where $A=a / \sqrt{h}=2 /(3 \pi b \sqrt{h})$. Thus, the total jump of the adiabatic invariant can be written as

$$
\Delta J=\lim _{N \rightarrow \infty}\left(I_{-1}-I_{N}\right)=-A(\varepsilon \Theta)^{3 / 2} f(\xi)
$$

where

$$
f(\xi)=\frac{3}{2} \lim _{N \rightarrow \infty} \sum_{i=0}^{N}(\xi+i)^{1 / 2}-\frac{3}{4}(\xi+N)^{1 / 2}-(\xi+N)^{3 / 2} .
$$

The function $f(\xi)$ can be written in the integral form ${ }^{17}$

$$
f(\xi)=\frac{3}{4 \sqrt{\pi}} \int_{0}^{\infty} \frac{d t}{t^{3 / 2}}\left(\frac{1}{2}-\xi+\frac{1}{t}-\frac{\exp (-\xi t)}{1-e^{-t}}\right) .
$$

The final dimensional expression for the jump $\Delta J$ is

$$
\Delta J=-A(\varepsilon \Theta)^{3 / 2} f(\xi),
$$

where $\Delta J, A$, and $\Theta$ are written in dimensional form (we take into account that $\sqrt{2 h}=1$ when $h_{0}$ is equal to the initial value of energy $H$ )

$$
\begin{aligned}
& \Theta=-\frac{\pi c\left(2 h_{0}\right)^{3 / 2} \sqrt{m}}{e B_{0}^{2}} \frac{\partial B_{0}}{\partial s} \\
& A=\frac{2}{3 \pi} \frac{m c}{e B_{0} \sqrt{h_{0}}} .
\end{aligned}
$$

Equation (32) shows that $\Delta J \sim \varepsilon^{3 / 2}$. The variable $\xi$ changes significantly in case of small variations of initial particle position far from the position of taking off. Thus, $\xi$ is traditionally considered as a quasi-random variable with uniform distribution within the range $(0,1)$ (see Ref. 32$)$.

\section{NUMERICAL SIMULATIONS}

To verify Eq. (37), we numerically integrate trajectories of charged particles interacting with the boundary. Equation (37) shows that $\Delta J$ does not depend on the boundary curvature $k(s)$; thus, we restrict our consideration by a simplified system with the straight boundary. We introduce Cartesian coordinates $(x, y)$ and conjugate momenta $\left(p_{x}, p_{y}\right)$ for the dimensionless Hamiltonian

$$
H=\frac{1}{2} p_{x}^{2}+\frac{1}{2}\left(p_{y}-b(\varepsilon y) x\right)^{2}
$$

where $b(\varepsilon y)=1+(\varepsilon y)^{2}$ for $x>0$, and energy $h=1 / 2$. The improved adiabatic invariant $J$ coincides with $I$ at the boundary $x=0$; thus, we use $I$ defined at $x=0$ instead of calculation of function $u$.

An example of the particle trajectory in this system is shown in Fig. 2. The particle moves along the boundary $x=0$ reflecting from it and rotating in the magnetic field. This motion corresponds to the increase of $|y|$, i.e., the particle drifts toward the negative $y$ values. Due to the increase of $b$ with $|y|$, the particle Larmor radius becomes smaller and the distance between the boundary and the center of the Larmor circle becomes larger during the drift along the boundary. Around $y \approx-100$, the particle takes off from the boundary. This moment is shown in detail in the middle panel of Fig. 2. After taking off from the boundary, the
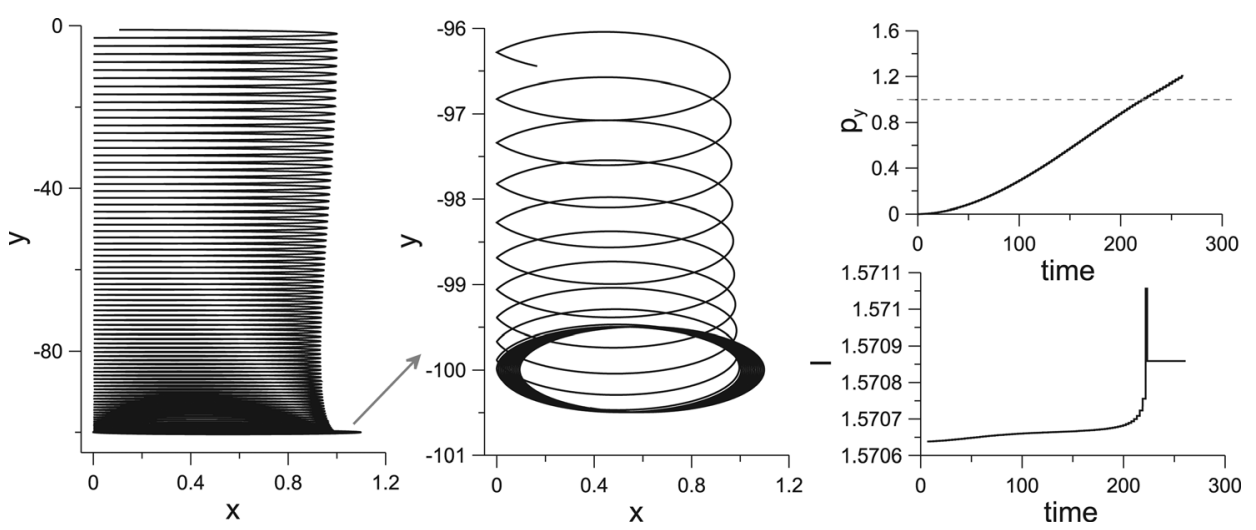

FIG. 2. The particle trajectory in the $(x, y)$ plane (left panel) and the moment of particle taking off (middle panel) are shown for system (39) with $\varepsilon=10^{-2}$. Right panels show $p_{y}$ and $I$ profiles along the trajectory. 

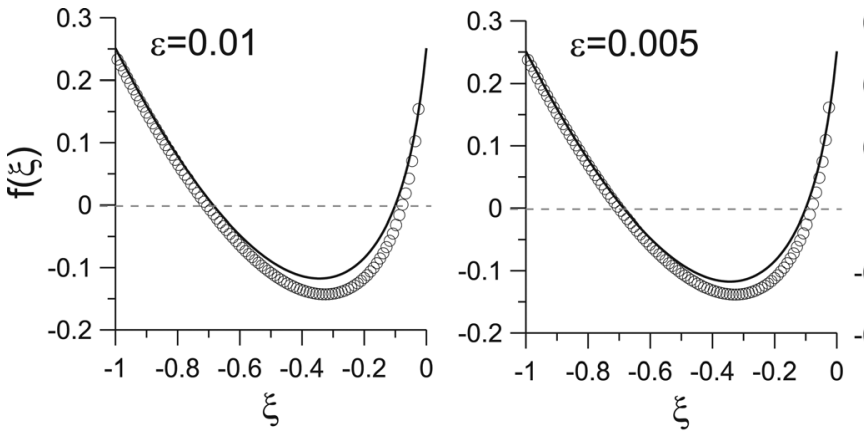

particle starts rotating around magnetic field without collisions with the boundary. This rotation is much faster than the drift along the boundary, while the boundary drift is much faster than the gradient drift of particles far from the boundary. Thus, the particle trajectory looks like a sequence of circles slightly shifted one relative to other (see the middle panel of Fig. 2). The moment of taking off corresponds to $p_{y}=1$ (i.e., $\delta=1-p_{y} / \sqrt{2 h}=0$ ). We show both profiles of $p_{y}$ and the adiabatic invariant $I$ to demonstrate that $I$ starts changing before the $p_{y}=1$ point, while for $p_{y}>1$ the adiabatic invariant is constant with a high accuracy. This change of the adiabatic invariant between the initial value and final (for $p_{y}>1$ ) value is the jump of $J$.

To check the dependence of $\Delta J$ on the small parameter $\varepsilon$ and on quasi-random variable $\xi$, we compare numerical results and the analytical expression for $\Delta J$. To this end, we choose three values of $\varepsilon$, generate an ensemble of $10^{6}$ particles with different $\xi$, and trace these particles backward and forward in time. Then, for each trajectory, we determine the jump of the adiabatic invariant and normalize it on the coefficient $-A(\varepsilon \Theta)^{3 / 2}$. For trajectories with the same $\xi$, we calculate the value of $f$ as a ratio of $\Delta J$ and $-A(\varepsilon \Theta)^{3 / 2}$ (see Eq. (37)). Comparison of such numerical functions $f(\xi)$ and integral expression (36) is shown in Fig. 3. One can see that for small enough $\varepsilon$ the numerical calculations describe analytical expressions quite well.

\section{DISCUSSION AND CONCLUSIONS}

The violation of adiabaticity described by Eq. (37) is related to a non-smooth dependence of the period of oscillations on energy near the separatrix: $T \approx T_{\text {sep }}-3 \pi a \sqrt{\kappa}$, Eq. (20). For separatrix crossings in smooth Hamiltonian systems considered in Refs. 9, 32, 33, and 46, the period has a logarithmic singularity on a separatrix, $T \sim \ln \kappa$. The corresponding jump in adiabatic invariant is $\Delta J \sim \varepsilon$. In our case, the singularity is weaker, $\sim \sqrt{\kappa}$, what results in a smaller jump: $\Delta J \sim \varepsilon^{3 / 2}$.

Besides the general theoretical interest, obtained results can be important for description of processes in cosmic plasma systems. Equation (37) shows that the jump of the adiabatic invariance in the system with strong magnetic field and impacts is about $\sim \varepsilon^{3 / 2}$. The amplitude of this jump is significantly larger than the rate of the magnetic moment change in system with strong magnetic field without a change of type of the particle motion, $\Delta J \sim \exp (-1 / \varepsilon)$ (see Refs. 14 and 47). There are several space plasma systems where magnetic field configuration assumes the billiard-like motion with the change of type of motion (drift along the boundary and gyrorotation at some distance from the boundary). These are magnetic structures with a local minimum of the magnetic field inside some volume and the strong magnetic field outside it (e.g., plasmoids, ${ }^{40,45}$ magnetic holes, ${ }^{5,24,48}$ etc.). The particle (electron) motion inside such structures resembles the billiard motion. Thus, our theory predicts that for a time interval $\Delta t \sim 1 / \varepsilon$ a change of the magnetic moment for such particles is about $\varepsilon^{3 / 2}$. The averaged jump $\langle\Delta J\rangle_{\xi}$ is equal to zero, thus, the rate of the adiabatic invariant diffusion is determined as $\left\langle(\Delta J)^{2}\right\rangle_{\xi} / \Delta t \sim \varepsilon^{4}$. As a result, for a time interval $\sim \varepsilon^{-4}$ all particles inside such magnetic structures should be significantly mixed due to the destruction of initial magnetic momenta.

To conclude, we have derived the equation for the rate of adiabatic invariance destruction $\Delta J(33,37)$ for magnetic billiards. We have shown that $\Delta J$ does not depend on the boundary curvature $k(s)$ as well as on the magnetic field inhomogeneity across the boundary $\partial B / \partial r$. The scaling of $\Delta J \sim \varepsilon^{3 / 2}$ and dependence of $\Delta J$ on $\xi$ are similar with the scaling and dependence $\Delta J(\xi)$ derived before for a potential system with impacts. ${ }^{17}$ Thus, there is a certain universality of the process of adiabaticity destructions in the system with impacts. However, in contrast to the system, ${ }^{17}$ in the billiard with the inhomogeneous magnetic field, many takes off from the boundary (i.e., many jumps of the adiabatic invariant) result in a stochastization of motion in large phase space domain. $^{34}$

\section{ACKNOWLEDGMENTS}

The work of A.V.A. was supported by the Russian Foundation for Basic Research (Project No. 13-01-00251). The work of A.I.N. was supported by the Council of the President of the Russian Federation for Support of Young Scientists and Leading Scientific Schools (Project No. NSh2964.2014.1).

${ }^{1}$ M. Aichinger, S. Janecek, and E. Räsänen, "Billiards in magnetic fields: A molecular dynamics approach," Phys. Rev. E 81(1), 016703 (2010).

${ }^{2}$ A. I. Akhiezer, N. F. Shul'ga, V. I. Truten', A. A. Grinenko, and V. V. Syshchenko, "Dynamics of high-energy charged particles in straight and bent crystals," Phys. Usp. 38, 1119-1145 (1995).

${ }^{3}$ A. I. Akhiezer, V. I. Truten', and N. F. Shul'ga, "Dynamic chaos in the motion of charged particles through a crystal," Phys. Rep. 203, 289-343 (1991).

${ }^{4}$ V. I. Arnold, V. V. Kozlov, and A. I. Neishtadt, "Mathematical aspects of classical and celestial mechanics," in Dynamical Systems. III. 
Encyclopaedia of Mathematical Sciences, 3rd ed. (Springer-Verlag, New York, 2006).

${ }^{5}$ M. A. Balikhin, R. Z. Sagdeev, S. N. Walker, O. A. Pokhotelov, D. G. Sibeck, N. Beloff, and G. Dudnikova, "THEMIS observations of mirror structures: Magnetic holes and instability threshold," Geophys. Res. Lett. 36, 3105, doi:10.1029/2008GL036923 (2009).

${ }^{6}$ S. Bernardi, J. S. Hansen, F. Frascoli, B. D. Todd, and C. P. Dettmann, "Ergodicity of a single particle confined in a nanopore," J. Stat. Phys. 148, 1156-1169 (2012).

${ }^{7}$ W. Breymann, Z. Kovács, and T. Tél, "Chaotic scattering in the presence of an external magnetic field," Phys. Rev. E 50, 1994-2006 (1994).

${ }^{8}$ L. A. Bunimovich and Y. G. Sinai, "Statistical properties of Lorentz gas with periodic configuration of scatterers," Commun. Math. Phys. 78, 479-497 (1981).

${ }^{9}$ J. R. Cary, D. F. Escande, and J. L. Tennyson, "Adiabatic-invariant change due to separatrix crossing," Phys. Rev. A 34(5), 4256-4275 (1986).

${ }^{10} \mathrm{G}$. Casati and T. Prosen, "Mixing property of triangular billiards," Phys. Rev. Lett. 83, 4729-4732 (1999).

${ }^{11} \mathrm{G}$. Casati and T. Prosen, "Time irreversible billiards with piecewisestraight trajectories," Phys. Rev. Lett. 109(17), 174101 (2012).

${ }^{12} \mathrm{~N}$. Chernov, "Decay of correlations and dispersing billiards," J. Stat. Phys. 94, 513-556 (1999).

${ }^{13}$ N. Chernov, A. Korepanov, and N. Simányi, "Stable regimes for hard disks in a channel with twisting walls," Chaos 22(2), 026105 (2012).

${ }^{14}$ B. V. Chirikov, Particle Dynamics in Magnetic Traps, Reviews of Plasma Physics, Vol. 13, 1st ed. (Consultants Bureau, New York, 1987).

${ }^{15} \mathrm{M}$. Eichengrün, W. Schirmacher, and W. Bregmann, "Quantum chaotic scattering with a mixed phase space: The three-disk billiard in a magnetic field," Phys. Rev. E 61, 382 (2000).

${ }^{16}$ A. Góngora-T, J. V. José, and S. Schaffner, "Classical solutions of an electron in magnetized wedge billiards," Phys. Rev. E 66(4), 047201 (2002).

${ }^{17}$ I. Gorelyshev and A. Neishtadt, "Jump in adiabatic invariant at a transition between modes of motion for systems with impacts," Nonlinearity 21, 661-676 (2008).

${ }^{18}$ B. Gutkin, "Hyperbolic magnetic billiards on surfaces of constant curvature," Commun. Math. Phys. 217, 33-53 (2001).

${ }^{19}$ E. Gutkin, "Billiard dynamics: An updated survey with the emphasis on open problems," Chaos 22(2), 026116 (2012).

${ }^{20}$ K. Hornberger and U. Smilansky, "Magnetic edge states," Phys. Rep. 367, 249-385 (2002).

${ }^{21}$ A. Kamor, F. Mauger, C. Chandre, and T. Uzer, "Annular billiard dynamics in a circularly polarized strong laser field," Phys. Rev. E 85(1), 016204 (2012).

${ }^{22}$ S. Kawabata and K. Nakamura, "Ballistic conductance fluctuation and quantum chaos in Sinai billiard,” J. Phys. Soc. Jpn. 66, 712 (1997).

${ }^{23}$ Z. Kovács, "Orbit stability in billiards in magnetic field," Phys. Rep. 290, 49-66 (1997).

${ }^{24}$ E. A. Kuznetsov, T. Passot, and P. L. Sulem, "Dynamical model for nonlinear mirror modes near threshold," Phys. Rev. Lett. 98(23), 235003 (2007).

${ }^{25}$ L. D. Landau and E. M. Lifshitz, The Classical Theory of Fields, Course of Theoretical Physics, Vol. 2 (Pergamon Press, 1971).

${ }^{26}$ J. F. Laprise, A. Hosseinizadeh, J. Lamy-Poirier, R. Zomorrodi, J. Kröger, and H. Kröger, "Universality in level spacing fluctuations of a chaotic optical billiard," Phys. Lett. A 374, 2000-2004 (2010).

${ }^{27}$ E. D. Leonel, M. W. Beims, and L. A. Bunimovich, "Introduction to focus issue: Statistical mechanics and billiard-type dynamical systems," Chaos 22(2), 026101 (2012).

${ }^{28}$ E. D. Leonel and L. A. Bunimovich, "Suppressing Fermi acceleration in two-dimensional driven billiards," Phys. Rev. E 82(1), 016202 (2010).
${ }^{29}$ E. D. Leonel, P. V. McClintock, and J. K. da Silva, "Fermi-Ulam accelerator model under scaling analysis," Phys. Rev. Lett. 93(1), 014101 (2004).

${ }^{30} \mathrm{P}$. J. J. Luukko and E. Räsänen, "Imaginary time propagation code for large-scale two-dimensional eigenvalue problems in magnetic fields," Comput. Phys. Commun. 184, 769-776 (2013).

${ }^{31}$ C. M. Marcus, A. J. Rimberg, R. M. Westervelt, P. F. Hopkins, and A. C. Gossard, "Conductance fluctuations and chaotic scattering in ballistic microstructures," Phys. Rev. Lett. 69, 506-509 (1992).

${ }^{32} \mathrm{~A}$. Neishtadt, "On the change in the adiabatic invariant on crossing a separatrix in systems with two degrees of freedom," J. Appl. Math. Mech. 51, 586-592 (1987).

${ }^{33}$ A. I. Neishtadt, "Change of an adiabatic invariant at a separatrix," Sov. J. Plasma Phys. 12, 568-573 (1986).

${ }^{34}$ A. I. Neishtadt and A. V. Artemyev, "Destruction of adiabatic invariance for billiards in a strong nonuniform magnetic field," Phys. Rev. Lett. 108(6), 064102 (2012).

${ }^{35}$ T. G. Northrop, The Adiabatic Motion of Charged Particles (Interscience Publishers, John Wiley and Sons, New York-London-Sydney, 1963).

${ }^{36}$ D. F. M. Oliveira and E. D. Leonel, "In-flight and collisional dissipation as a mechanism to suppress Fermi acceleration in a breathing Lorentz gas," Chaos 22(2), 026123 (2012).

${ }^{37}$ R. Pérez-Pascual, B. M. Rodríguez-Lara, and R. Jáuregui, "Chaotic dynamics of thermal atoms in labyrinths created by optical lattices," J. Phys. B: At. Mol. Phys. 44(3), 035303 (2011).

${ }^{38}$ A. Rapoport and V. Rom-Kedar, "Chaotic scattering by steep repelling potentials," Phys. Rev. E 77(1), 016207 (2008).

${ }^{39}$ M. Robnik and M. V. Berry, "Classical billiards in magnetic fields," J. Phys. A 18, 1361-1378 (1985).

${ }^{40}$ A. S. Sharma, R. Nakamura, A. Runov, E. E. Grigorenko, H. Hasegawa, M. Hoshino, P. Louarn, C. J. Owen, A. Petrukovich, J.-A. Sauvaud, V. S. Semenov, V. A. Sergeev, J. A. Slavin, B. U. Ã-. Sonnerup, L. M. Zelenyi, G. Fruit, S. Haaland, H. Malova, and K. Snekvik, "Transient and localized processes in the magnetotail: A review," Ann. Geophys. 26, 955-1006 (2008).

${ }^{41}$ Y. G. Sinai, "Dynamical systems with elastic reflections," Russ. Math. Surv. 25, 137-189 (1970).

${ }^{42}$ H.-J. Stöckmann and J. Stein, “'Quantum' chaos in billiards studied by microwave absorption," Phys. Rev. Lett. 64, 2215-2218 (1990).

${ }^{43}$ T. Tasnádi, "The behavior of nearby trajectories in magnetic billiards," J. Math. Phys. 37, 5577-5598 (1996).

${ }^{44}$ T. Tasnádi, "Hard chaos in magnetic billiards (on the hyperbolic plane)," J. Math. Phys. 39, 3783-3804 (1998).

${ }^{45}$ W.-L. Teh, S. Eriksson, B. U. Ö. Sonnerup, R. Ergun, V. Angelopoulos, K.-H. Glassmeier, J. P. McFadden, and J. W. Bonnell, "THEMIS observations of a secondary magnetic island within the Hall electromagnetic field region at the magnetopause," Geophys. Res. Lett. 37, 21102, doi:10.1029/ 2010GL045056 (2010).

${ }^{46} \mathrm{~A}$. V. Timofeev, "On the problem of constancy of the adiabatic invariant during change of the type of motion," Sov. J. JETP 75, 1303-1308 (1978).

${ }^{47}$ R. K. Varma, "Classical and macroquantum dynamics of charged particles in a magnetic field," Phys. Rep. 378, 301-434 (2003).

${ }^{48}$ T. L. Zhang, W. Baumjohann, C. T. Russell, L. K. Jian, C. Wang, J. B. Cao, M. Balikhin, X. Blanco-Cano, M. Delva, and M. Volwerk, "Mirror mode structures in the solar wind at 0.72 AU," J. Geophys. Res. 114, 10107, doi:10.1029/2009JA014103 (2009).

${ }^{49}$ V. Zharnitsky, "Quasiperiodic motion in the Hamiltonian systems of the billiard type,” Phys. Rev. Lett. 81, 4839-4842 (1998).

${ }^{50}$ V. Zharnitsky, "Invariant tori in Hamiltonian systems with impacts," Commun. Math. Phys. 211, 289-302 (2000). 